\title{
Molecular Pathogenesis of Alzheimer's Disease
}

\author{
An Expert Interview with Lars Tjernberg and Sophia Schedin Weiss
}

Karolinska Institutet, Stockholm, Sweden

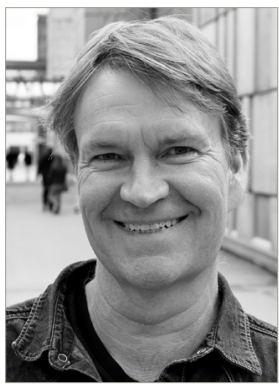

\section{Lars Tjernberg}

Lars Tjernberg, PhD, is a group leader and an Associate Professor at the Department of Neurobiology, Care Sciences and Society, Center for Alzheimer Research, Division of Neurogeriatrics, Karolinska Institutet, Stockholm, Sweden. Lars has a Master's degree in analytical chemistry and spent his first active years in the pharmaceutical industry. He received his PhD from Karolinska Institutet in 1998, with a thesis about Alzheimer's disease and the amyloid $\beta$-peptide $(A \beta)$. He continued his work on A $\beta$ during his postdoc at the Picower Institute for Medical Research in New York. Returning to Sweden and Karolinska Institutet, he expanded his work to the enzymes involved in $A \beta$ production, and how these can be regulated. His focus is now to study $A \beta$ in neurons by novel technology, and based on these results develop novel treatment strategies.

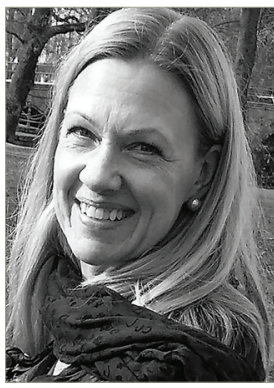

\section{Sophia Schedin Weiss}

Sophia Schedin Weiss, PhD, is an Associate Professor at the Department of Neurobiology, Care Sciences and Society, Center for Alzheimer Research, Division of Neurogeriatrics, Karolinska Institutet, Stockholm, Sweden. Sophia Schedin Weiss studied chemistry at Stockholm University and received her PhD in biochemistry at Stockholm University in 1998. She did a postdoc at the Swedish University for Agricultural Sciences, Uppsala, specializing in protein-protein and protein-glycan interactions. She obtained her docentur in medical biochemistry at Uppsala University in 2008. Since 2010 she is enrolled in research related to Alzheimer disease at Karolinska Institutet. Her main focus areas are to study the subcellular location of A $\beta$ formation in neurons using super-resolution microscopy and identify glycan biomarkers for Alzheimer's disease and other neurodegenerative disorders.

\section{Keywords}

Alzheimer's disease, amyloid $\beta$-peptide

Disclosures: Lars Tjernberg and Sophia Schedin Weiss have no conflicts of interest to declare in relation to the article.

Review process: This is an expert interview and, as such, has not undergone the journal's standard peer review process.

Acknowledgments: Medical writing assistance was provided by Katrina Mountfort of Touch Medical Media.

Authorship: All named authors meet the International Committee of Medical Journal Editors (ICMJE) criteria for authorship of this manuscript, take responsibility for the integrity of the work as a whole, and have given final approval to the version to be published.

Received: January 7, 2019

Accepted: January 18, 2019

Citation: US Neurology. 2019;15(1):14-6

Corresponding Author: Sophia Schedin Weiss, Department of NVS, Center for Alzheime Research, Division of Neurogeriatrics, Karolinska Institutet, 17164 Solna, Sweden.

Support: No funding was received in the publication of this article.
A Izheimer's disease was first described in 1906, and yet we have still not fully elucidated the pathogenesis of the condition. It is known that the disease is characterized by the polymerization of amyloid $\beta$-peptide $(A \beta)$, leading to the formation of plaques., ${ }^{1,2}$ The cascade of events initiated by $A \beta$ polymerization eventually leads to progressive neurodegeneration and brain dysfunction. However, we have not yet established how and exactly where in the cell $A \beta$ is generated from amyloid precursor protein (APP) and where the polymerization takes place. One technical challenge is the fact that most subcellular compartments cannot be spatially resolved by traditional confocal light microscopy. New technological advances such as super-resolution microscopy have enabled us to visualize the location of $A \beta$ and $\gamma$-secretase, the enzyme complex that liberates A $\beta$ from its precursor APP, in neurons.

In an expert interview, Lars Tjernberg and Sophia Schedin Weiss discuss their microscopy studies that are elucidating the molecular pathways that underline Alzheimer's disease. ${ }^{3-5}$ 

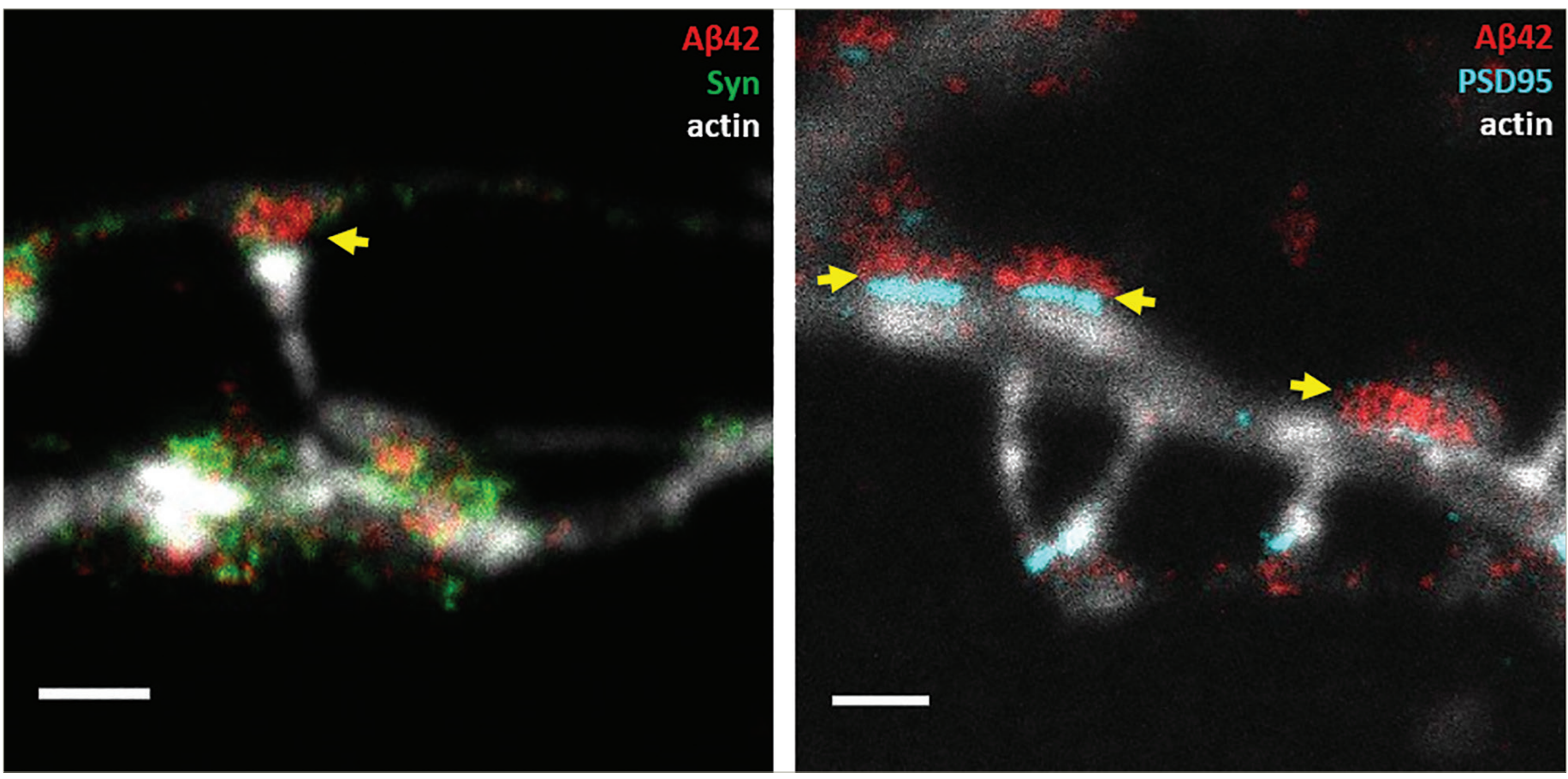

Stimulated Emission Depletion (STED) microscopy was used to image synapses of immuno-labeled primary hippocampal neurons. Left: Aß42 (red) and the pre-synaptic marker synaptophysin (syp, green). Right: A 42 (red) and the post-synaptic density (cyan). Neuronal structure is shown by actin in a confocal channel (light grey) in both images. Scale bars, $1 \mu \mathrm{m}$. Yellow arrows point at A 42 vesicles in the pre-synapse. In the right image, the distance between the post-synapse and the pre-synaptic A 42 vesicles is around $25 \mathrm{~nm}$. $A \beta=$ amyloid $\beta$-peptide

\section{Q. What is your view on the pathogenesis of Alzheimer's disease?}

LT: The most important factor in Alzheimer's disease is a small piece of protein - the $A \beta$ - that comes in different lengths. One peptide with 42 amino acids has been found to be toxic in laboratory studies with cell lines and in animal studies. ${ }^{6,7}$ Deposits of this peptide as amyloid plaques were first found in the human brain over 100 years ago. Now we have a clear understanding on how $A \beta$ is produced. The final cleavage reaction that produces A $\beta$ from its APP is mediated by $\gamma$-secretase. A number of clinical trials have aimed to stop $A \beta$ production by inhibiting this $\gamma$-secretase enzyme complex.8,9 Unfortunately, $\gamma$-secretase has around 100 different substrates and therefore we need to be more specific in inhibiting A $\beta$ production.

\section{Q. Why did you initiate these studies?}

LT: We wanted to find out exactly where in the cell $A \beta$ is produced from its precursor. To achieve this, we wanted to use neurons rather than cell lines that are used in many other studies. Neurons were obtained from mice and cultured in vitro, allowing them to form synapses. When examining these cells under the microscope we discovered that $\gamma$-secretase is concentrated at synapses, both at the pre-synaptic and post-synaptic sides. ${ }^{3}$ It is located in small, spherical structures called vesicles on both sides and at the postsynaptic side it is also located at the cell membrane. When we repeated these studies to locate $A \beta$, we found that it was only located at the presynaptic side in similar structures, in many cases containing a protein called synaptophysin, which means that these are vesicles involved in synaptic transmission.

\section{Q. How can you obtain such impressive resolution?}

ssw: Light microscopes are commonly used to look at cells and tissues. Fluorescence microscopes are a type of light microscope that take advantage of the fact that certain molecules, known as fluorophores, excited by light of a certain wavelength, can emit light of another wavelength. By using fluorophores coupled to compounds that bind specifically to certain molecules, we can visualize specific components in cells. Since light propagates as waves, the light hitting the sample diffracts. Thus, even using an objective lens, we cannot focus the light to more than half the wavelength, which is usually around $200-350 \mathrm{~nm}$.

Particles smaller than this cannot be resolved, however, scientists have discovered that fluorophores, which emit light, can be switched into dark states. This fact has been taken advantage of to overcome resolution limitations caused by the diffraction. One way to achieve this is to turn molecules dark by stimulated emission. ${ }^{10}$ This can be achieved in specialized laser scanning microscopes by overlaying the excitation laser light with a depletion laser light in a donut-shaped pattern at the edges of the fluorescent spot. This switches off the fluorescence at the edges of the spot, minimizing the size of the spot, resulting in improved resolution, as if we could paint using a thinner brush.

Another principle used to increase resolution is known as single molecule localization microscopy. ${ }^{11,12}$ With this technique, the fact that certain fluorophores under certain circumstances can switch between fluorescent and dark states is used, which results in stochastic blinking of the sample 
when illuminated with high power laser light. By using very rapid cameras it is possible to produce a series of images where only a few fluorophores are turned on in each image. These can be resolved from each other and the exact center of each fluorescent spot can be determined. By adding together the data from such a series of images, a super-resolved image can be reconstructed.

These two examples represent ways to achieve superior resolution in different types of fluorescence microscopes. We can achieve at least tenfold improved resolution compared with confocal microscopes. This makes it possible for us to visualize small structures in the cells where Alzheimer's disease pathology takes place, such as the small vesicles at the synapses.

LT: To give you some idea of the dimensions we are looking at, the synaptic vesicles are around $40 \mathrm{~nm}$ in diameter and the synaptic cleft, i.e., the distance between the pre- and post-synapse, is around $20 \mathrm{~nm}$. With the traditional confocal microscope we have a resolution of around $200 \mathrm{~nm}$. With our system, we have a resolution of $10-30 \mathrm{~nm}$, so this technique is perfect to resolve the structures that we are studying.

Ssw: Although many previous studies have been performed on $A \beta$ and $\gamma$-secretase, we have not seen any other publication where they have used these super-resolution microscopy techniques to image $\gamma$-secretase and $A \beta$ in neurons.

LT: I think we will see many more similar studies since these microscopes are becoming available to more and more scientists and people realize how much they can improve their studies by using these techniques.

\section{Q. What are the major findings of your investigations?}

LT: The major findings were that $\gamma$-secretase is located both presynaptically and post-synaptically, ${ }^{3}$ whereas $A \beta$ is only located at the presynapse, ${ }^{4}$ potentially in synaptic vesicles. When we see these well-defined structures containing $\gamma$-secretase and $A \beta$, we realize that they must be there for a reason and we should be very careful when we try to limit $A \beta$ production, the most popular treatment approach, in order to treat AD.
Since $\gamma$-secretase has so many substrates, treatment needs to be very specific. One possibility would be to target $\gamma$-secretase at the pre-synapse and leave the other side untouched.

\section{Q. How could your results help us treat Alzheimer's disease?}

LT: These results may be a starting point for thinking how to reach this particular pool of $A \beta$ at the pre-synapse and also that we should not reduce $A \beta$ production too much because it may have a physiological function. Our studies suggest that we can be more specific in our treatment approaches.

SSW: Since we identified different types of vesicles that contain $A \beta$ in the neurons, one of our aims is to identify the nature of these vesicles.

LT: We think that there are other types of vesicles and we want to define the differences between them. We also want to try some pre-commercial microscopes that have super-resolution in all three dimensions. This will allow us to make three-dimensional, super-resolved images and thus characterize the vesicles even better.

\section{Q. Where will you go from here?}

ssw: We have observed A 342 in different types of vesicles in the neurons. Some of these are synaptic vesicles, but we have also seen other types of vesicles that contain A $\beta 42$. We aim to identify the nature of these vesicles using more super-resolution studies. We also aim to set up live cell imaging experiments so we will be able to follow exactly where in the cell $A \beta$ is formed. This will allow us to view how $A \beta$ is transported inside the cells and where it starts to aggregate, as well as how it can be transferred from cell to cell. All these studies will be performed in neurons with super-resolution.

LT: We also have ways of looking at $A \beta$ when it polymerizes. $A \beta$ is produced as a monomer and initially it is not toxic. When it starts to polymerize, it forms toxic oligomers. Combining the super-resolution techniques that we use with a novel $A \beta$ labeling technique that we develop we will be able to follow the polymerization process in living neurons and elucidate exactly where it takes place.
1. Deane R, Bell RD, Sagare A, et al. Clearance of amyloid-beta peptide across the blood-brain barrier: implication for therapies in Alzheimer's disease. CNS Neurol Disord Drug Targets. 2009;8:16-30

2. Pena F, Gutierrez-Lerma A, Quiroz-Baez R, et al. The role of betaamyloid protein in synaptic function: implications for Alzheimer's disease therapy. Curr Neuropharmacol. 2006;4:149-63.

3. Schedin-Weiss S, Caesar I, Winblad B, et al. Super-resolution microscopy reveals gamma-secretase at both sides of the neuronal synapse. Acta Neuropathol Commun. 2016;4:29.

4. Yu Y, Jans DC, Winblad B, et al. Neuronal $A \beta 42$ is enriched in small vesicles at the presynaptic side of synapses. Life Sci Alliance. 2018;1:e201800028.

5. Schedin-Weiss S, Inoue M, Teranishi Y, et al. Visualizing active enzyme complexes using a photoreactive inhibitor for proximity ligation-application on $\gamma$-secretase. PLOS One. 2013;8:e63962.

6. Sasaguri $\mathrm{H}$, Nilsson P, Hashimoto $\mathrm{S}$, et al. APP mouse models for Alzheimer's disease preclinical studies. EMBO J. 2017;36:2473-87.

7. Reiss AB, Arain HA, Stecker MM, et al. Amyloid toxicity in Alzheimer's disease. Rev Neurosci 2018;29:613-27.

8. Medoro A, Bartollino S, Mignogna D, et al. Complexity and selectivity of $\gamma$-secretase cleavage on multiple substrates: consequences in Alzheimer's disease and cancer. J Alzheimers Dis. 2018;61:1-15.

9. Tan Y, Zhang Q, Wong SG, Hua Q. Anti-Alzheimer therapeutic drugs targeting $\gamma$-secretase. Curr Top Med Chem. 2016;16:549-57.

10. Hell SW. Far-field optical nanoscopy. Science. 2007;316:1153-8.

11. Rust MJ, Bates M, Zhuang X. Sub-diffraction-limit imaging by stochastic optical reconstruction microscopy (STORM). Nat Methods. 2006;3:793-5.

12. Betzig E, Patterson $G H$, Sougrat $R$, et al. Imaging intracellular fluorescent proteins at nanometer resolution. Science. 2006;313:1642-5. 